\title{
Development of hydroxyapatite-based nanomaterials to enhance biological response of osteoblast cells for clinical application
}

\author{
Jian $\mathrm{Li}^{1}$, Yaping Liu ${ }^{2}$, Kun $\mathrm{Li}^{3}$ and Haitao Meng ${ }^{1 *}$ \\ ${ }^{1}$ Department of Bone and Joint Surgery, ${ }^{2}$ Department of Endocrinology, Jining First People's Hospital, Jining City, 272100 , \\ ${ }^{3}$ Department of Gynaecology and Obstetrics, Affiliated Hospital of Shandong Academy of Medical Sciences, Jinan City, \\ Shandong Province, 250031, China
}

*For correspondence: Email: menghaitao1969@hotmail.com; Fax/Tel: +86 5372253431

\begin{abstract}
Purpose: To develop a novel chitosan/gelatin-hydroxyapatite (CGHaP) microspheres for evaluating the biological response of pre-osteoblast cells.

Methods: The microsphere was prepared by water-in-oil emulsion method. Cell proliferation was studied using AlamarBlue colorimetric assay and DAPI staining while alkaline phosphatase assay was carried out by colorimetric assay method. Chitosan microspheres as well as chitosan-hydroxyapatite microspheres was prepared and tested for biological response from MC3T3-E1 cell line.

Results: The results showed that CGHaP promotes MC3T3-E1 cell proliferation and spread on the surface of microspheres. The cells were clustered with more actin filaments and well-linked with neighbouring cells or adjacent cells when cultured in CGHaP microspheres whereas fewer cells were spread on chitosan $(\mathrm{CH})$ microspheres. CGHaP microspheres significantly $(p<0.05)$ promoted cell attachment, proliferation and extracellular matrix mineralization. CGHaP microspheres presented significantly $(p<0.02)$ higher calcium deposition $(0.5 \mathrm{ng})$ than $\mathrm{CH}$ microspheres $(0.28 \mathrm{ng})$. Specifically, CGHaP microspheres exhibited high ALP activity (8 units; 2-fold) compared to $\mathrm{CH}$ with 3 units, after 7 days of incubation. The results suggest that $\mathrm{CGHaP}$ possesses a great ability to facilitate bone ingrowth formation and possibility of good osteointegration in vivo.

Conclusion: The nanomaterial enhances the proliferation of pre-osteoblast cells in tissue engineering microspheres. The outcome of this study may have a major impact on the development of novel nanomaterials for bone tissue engineering.
\end{abstract}

Keywords: Chitosan/gelatin-hydroxyapatite, Osteoblast, Osteoinduction, Bone tissue engineering, Nanomaterials, Microspheres, Calcium nodule formation

Tropical Journal of Pharmaceutical Research is indexed by Science Citation Index (SciSearch), Scopus, International Pharmaceutical Abstract, Chemical Abstracts, Embase, Index Copernicus, EBSCO, African Index Medicus, JournalSeek, Journal Citation Reports/Science Edition, Directory of Open Access Journals (DOAJ), African Journal Online, Bioline International, Open-J-Gate and Pharmacy Abstracts

\section{INTRODUCTION}

Nanomaterial that can mimic the critical aspects of natural biological processes will be much suitable for the tissue engineering. In recent years, organic-inorganic hybrid nanomaterials have been center of research due to its remarkable applications in biomedical field $[1,2]$.
In this regard, hydroxyapatite (HA) $\left\{\mathrm{Ca}_{10}\left(\mathrm{PO}_{4}\right)_{6}(\mathrm{OH})_{2}\right\}$ is a biological active calcium phosphate ceramic which is used to mimic bone in orthopedic surgery [3,4]. The excellent biocompatibility, bioactivity and osteoconductivity make it a natural choice for bone applications. However, nanocomposite materials of HA needs to be developed for bone tissue applications. In 
search of environment friendly materials from natural source which can be efficiently combined with the HA, we have selected chitosan (CS) and gelatin $(G L)$ as a potential candidate due to its excellent properties such as biocompatibility, non- immunogenicity, non-cytotoxicity, mucus adhesion, and low cost $[5,6]$. CS/GL when combined with the HA is expected to bring much needed flexibility to the composite materials. Moreover, $\mathrm{CS} / \mathrm{GL}$ is a natural polysaccharide which is biodegradable under in vivo conditions (due to lysozyme) $[7,8]$. With the single aim of controlling the proliferation, differentiation and bone-forming activities, we have designed microsphere which has large surface area for the cell growth. It has been reported that proliferation of osteoblast cells and osteogenic differentiation is utmost important for the bone tissue regeneration and the materials which promotes such activities are osteogenic agents $[9,10]$.

To best of our knowledge, we are the first group to report the in vitro osteoblast cell response in chitosan/gelatin-hydroxyapatite (CGHaP) microspheres. In this study, chitosan microspheres as well as chitosan-hydroxyapatite microspheres was prepared and biological response of MC3T3-E1 cell was studied. Specifically, cell proliferation, cell spreading, bone forming ability, calcium content, as well as extracellular matrix mineralization was evaluated.

\section{EXPERIMENTAL}

\section{Materials}

Chitosan (CS) with degree of deacetylation (> 90 $\%$ ) was obtained as a kind gift from Shanghai Bio Life Science and Technology Ltd. Gelatin and hydroxyapatite was purchased from SigmaAldrich, China. All chemicals were used without further purification.

\section{Preparation of chitosan/gelatin-hydroxy- apatite (CGHaP) microsphere}

The CGHaP microsphere was preparing by water-in-oil emulsion method. In brief, 2:1 weight ratio of chitosan/gelatin was dissolved in $50 \mathrm{ml}$ of acetic acid $(0.1 \mathrm{~N})$ solution. A fixed concentration of $\mathrm{HaP}$ was added to this solution and sonicated for $10 \mathrm{~min}$ to form a homogenous dispersion of polymer and inorganic molecules. Similarly, microspheres with HaP were also prepared as a control to compare. The amine groups present in the chitosan and gelatin were crosslinked by the addition of $2.5 \%$ glutaraldehyde. The resulting microspheres were washed 5 - 6 times with acetone and rinsed with double distilled water. In addition, residual glutaraldehyde was neutralized by keeping the microsphere in $2 \%$ sodium bisulfide for $24 \mathrm{~h}$ and then followed by repeated washing with water. The microspheres were then lyophilized using an automated freeze dryer.

\section{Morphological characterization}

The morphology and proliferation of MC3T3-E1 cells in $\mathrm{CH}$ and $\mathrm{CGHaP}$ microspheres were evaluated by an optical microscope (Olympus, Germany). Earlier, MC3T3-E1 cells were incubated with respective microspheres for 4 days and after the designated time point, microspheres were washed, and fixed with $4 \%$ glutaraldehyde. After fixing, samples were rinsed with DI water and dehydrated with sequential incubations of 20,40,60, 80, 90 and $100 \%$ ethanol in DI water for $15 \mathrm{~min}$ at each concentration and observed under light microscope. Samples were sterilized with $70 \%$ ethanol and washed thoroughly with PBS prior to cell seeding.

\section{Cell proliferation}

The cell proliferation was studied using AlamarBlue colorimetric assay. Briefly, cells were seeded in a 6-well plate along with $\mathrm{CH}$ and $\mathrm{CGHaP}$ microspheres and incubated for 1,4 , and 7 days, respectively. After incubation, well plates were washed twice with PBS and incubated in $10 \%(\mathrm{v} / \mathrm{v})$ AlamarBlue in DMEM with $10 \%$ FBS for $2 \mathrm{~h}$ at $37^{\circ} \mathrm{C}$. The solution was transferred to a 96-well plate and fluorescence was read in $560 \mathrm{~nm}$ using a spectrophotometer (SpectraMax, USA).

\section{Mineralization assay}

Cells after 7 days incubation were washed and soaked in $1 \mathrm{M}$ acetic acid overnight. The solution was then collected and absorbance was measured at 610 as per the manufacturer's protocol of calcium assay kit (Jiancheng Bioengineering Institute, China).

\section{Cell proliferation by DAPI staining}

Same procedure was employed as mentioned under colorimetric assay method. In addition, cells were stained with 4,6-diamidino-2phenylindole (DAPI, $0.5 \mu \mathrm{g} / \mathrm{mL}$ ) for $15 \mathrm{~min}$. After DAPI staining, cells were fixed and observed under confocal microscopy (Nikon, Japan).

\section{Cell spreading analysis}

The spreading of MC3T3-E1 was studied by observing the cytoskeleton of cells. After 3 days 
of incubation, samples were washed to remove the unattached cells and cells on the microspheres were fixed with $4 \%$ PFA and permeabilized with $0.1 \%$ Triton $\mathrm{X}-100$ in PBS for $10 \mathrm{~min}$. The cells were then stained with rhodamine-phalloidin for $45 \mathrm{~min}$ and visualized under confocal laser scanning microscope (Nikon, Japan).

\section{Alkaline phosphatase assay}

Same procedure was employed as mentioned under colorimetric assay method. After 7 days of incubation, ALP activity was evaluated by determining the amount of $p$-nitrophenol using an ALP microplate test kit. The amount of ALP in the each case was measured at $405 \mathrm{~nm}$ using an automated microplate reader.

\section{Alizarin-red staining assay}

Cells after 7 days incubation were washed twice with PBS and fixed with $4 \%$ paraformaldehyde (PFA) for 20 min before being stained with $1 \%$ Alizarin-red solution for $1 \mathrm{~h}$. The samples were washed with distilled water and dried. For the quantitative analysis, orange dye was dissolved in $10 \%$ cetylpyridinium chloride (Sigma-Aldrich, China) in $10 \mathrm{~mm}$ sodium phosphate solution and the absorbance was measured at $620 \mathrm{~nm}$ using an automated microplate reader.

\section{Statistical analysis}

All the data are expressed as mean \pm standard deviation ( $S D, n=3)$, and were analyzed by oneway variance analysis (ANOVA) with SPSS 11.0 software. $P<0.05$ was considered statistically significant

\section{RESULTS}

\section{Cell spread}

The cell spreading was clearly visible in both the microspheres; however what is more interesting is the fact that MC3T3-E1 cells spread preferably better in the $\mathrm{CGHaP}$ microspheres with numerous pseudopods anchored to the microsphere surface and present as characteristic cuboidal morphology (Figure 1).

Cell spread was further confirmed by observing the cytoskeleton of cells after staining with rhodamine-phalloidin. CGHaP microspheres showed a confluent and multilayer morphology of cells (data not shown). Moreover, cells were clustered with more actin filaments and welllinked with neighbouring cells or adjacent cells. In contrast, cells in the $\mathrm{CH}$ microsphere presented fewer actin filaments and cells were present as monolayer. The cell spreading from rhodamine staining was consistent with the SEM based morphology analysis.

\section{Cell proliferation}

The cell proliferation capacity of MC3T3-E1 cells in $\mathrm{CH}$ and $\mathrm{CGHaP}$ microspheres were evaluated by AlamarBlue colorimetric assay. As seen (Figure 2), HaP containing microspheres significantly $(p<0.05)$ promoted the cell proliferation when compared with the microspheres which do not have HaP. This indicates that the incorporation of $\mathrm{HaP}$ into composite microspheres greatly encourages attached cells to proliferate, especially at day 7 , suggesting a high affinity of pre-osteoblasts (MC3T3-E1) for HaP.

\section{$\mathrm{CH}-\mathrm{M}$}
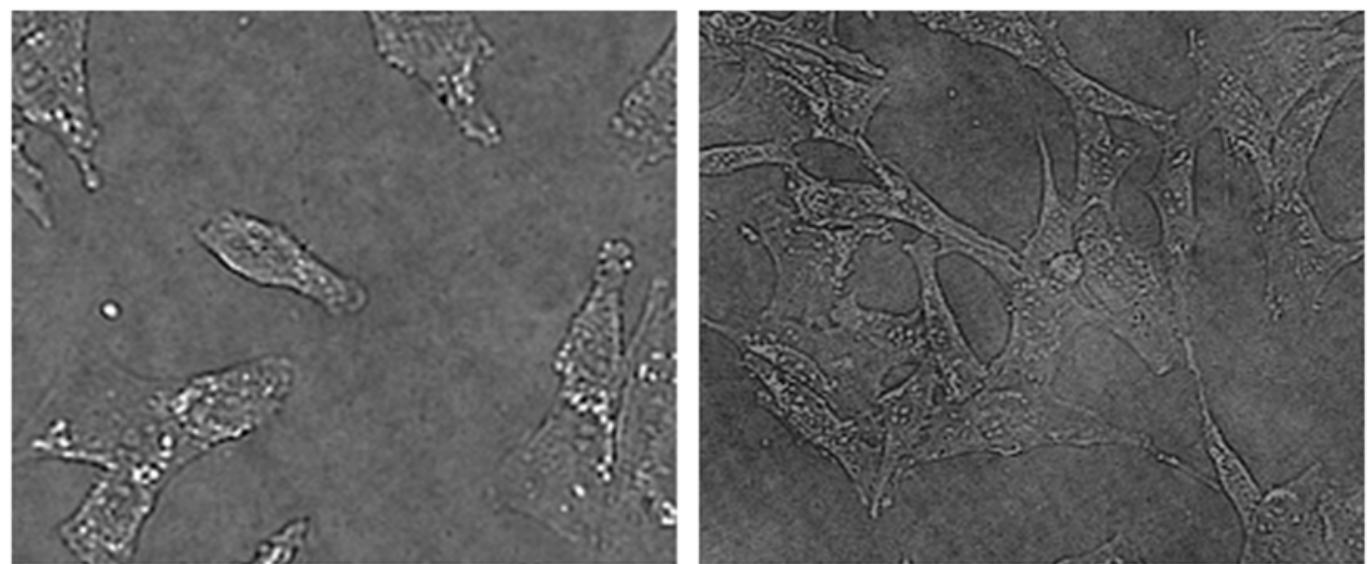

Figure 1: MC3T3-E1 cell spreading on chitosan microspheres and chitosan/gelatin-hydroxyapatite microspheres 


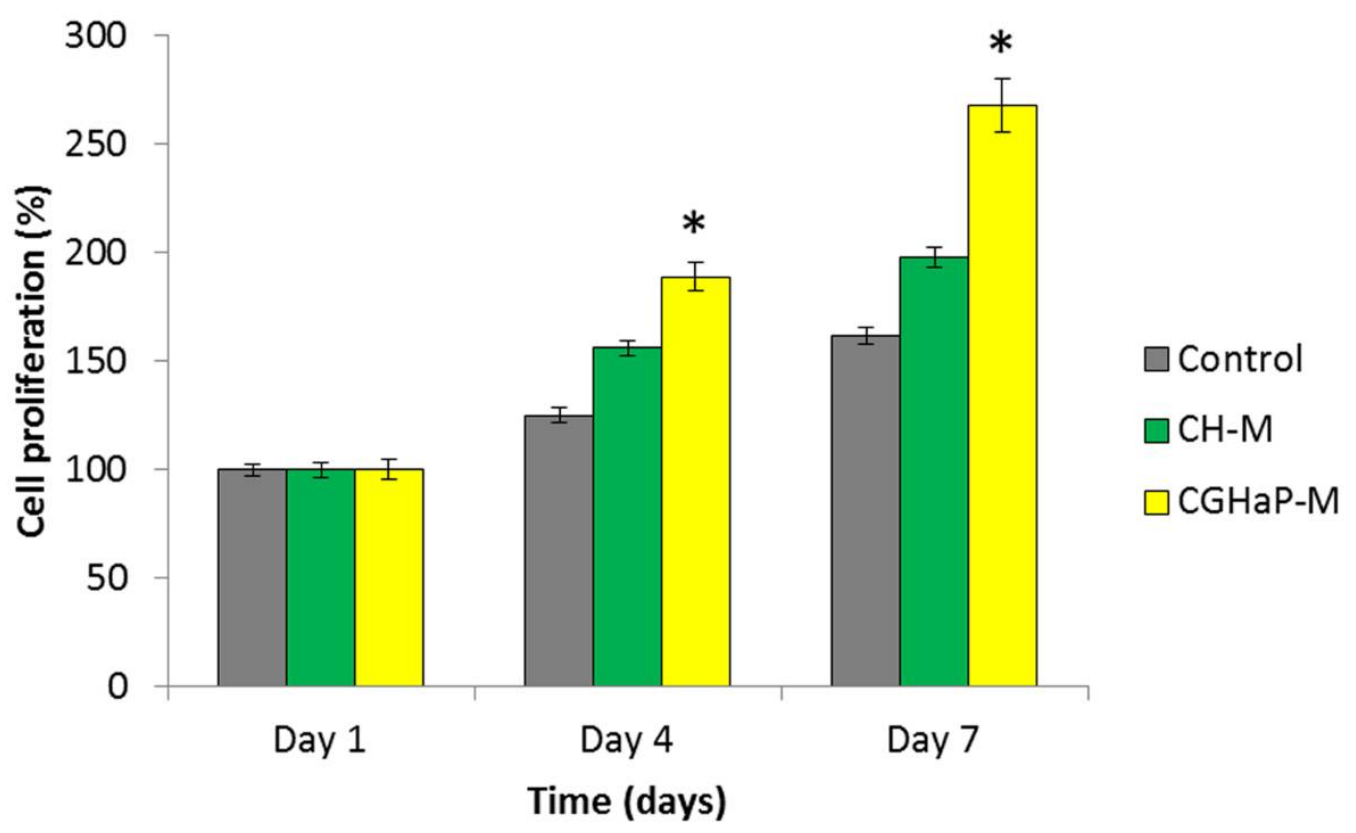

Figure 2: Cell proliferation analysis of MC3T3-E1 cells on chitosan microspheres and chitosan/gelatinhydroxyapatite microspheres after 7 days of incubation; ${ }^{*} p<0.05$ is the statistical difference between CGHaP-M and $\mathrm{CH}-\mathrm{M}$
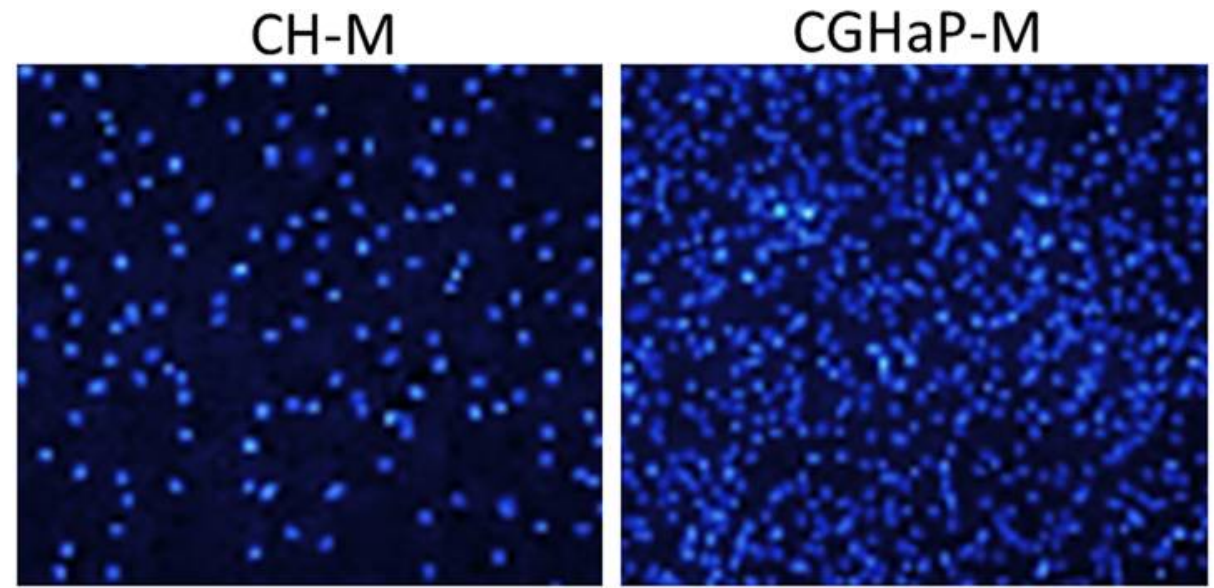

Figure 3: DAPI staining of MC3T3-E1 cell after 7 days of incubation

The cell proliferation was further confirmed by DAPI staining (Figure 3 ). The results clearly showed that cells grown on HaP microspheres exhibited remarkable blue fluorescence corresponding to the high cell proliferation.

\section{Extracellular matrix mineralization}

The calcium content from each group was quantified to evaluate the calcium deposition. The CGHaP microspheres presented the significantly $(p<0.02)$ higher calcium deposition $(0.5 \mathrm{ng})$ than $\mathrm{CH}$ microspheres $(0.28 \mathrm{ng})$. The remarkably calcium secretion was attributed to the presence of $\mathrm{HaP}$ in the medium and its nanoscale features.

\section{ALP staining}

Similarly, CGHaP microspheres showed stronger induction of ALP than compared to that of $\mathrm{CH}$ microspheres. Consistently, CGHaP microspheres showed a high ALP activity (8 unit; 2-fold) while $\mathrm{CH}$ showed lesser ALP activity (3 unit) after 7 days incubation.

\section{Alizarin-red staining}

Consistent with the ALP staining analysis, CGHaP microspheres showed a stronger and clearer Alizarin-red staining after 7 days of incubation (Figure 5b). Approximately, 4-fold higher intensity $(p<0.02)$ was observed than compared to that of $\mathrm{CH}$ microspheres. It has 
been reported that Alizarin-red staining is an important technique to evaluate the calcium nodules which corresponds to extracellular mineralization. Based on the result, it is clear that presence of $\mathrm{HaP}$ promoted the formation of calcium nodule formation and could promote the eventual cell differentiation

\section{DISCUSSION}

In this paper, chitosan/gelatin-hydroxyapatite microsphere was fabricated as a novel nanomaterial to improve the clinical performance in case of bone regeneration. HaP containing microspheres would allow the cell proliferation in the in vivo conditions. This microsphere has unique advantage of loading with proteins or growth factor or suitable drugs. HaP is expected to increase the in vitro calcification and cell proliferation. The mean size of $\mathrm{CH}$ microsphere was $\sim 15 \mu \mathrm{m}$; however, addition of $\mathrm{HaP}$ along with gelatin increased the average size to $\sim 35$ $\mu \mathrm{m}$. This is mainly ascribed to an increase in the viscosity of the HA-containing gelatin/chitosan solution. The resulting increase in the mean diameters by HA incorporation was also reported in other research that dealt with the fabrication of HA-embedded polymeric microspheres.

The high spread of cells in $\mathrm{HaP}$ containing microsphere was attributed to the bone-loving property of MC3T3-E1 cells wherein they exhibited a flattened shape. In contrast, fewer cells were observed in $\mathrm{CH}$ microspheres due to the absence of bone elements (HaP). Generally, cell attached and followed by spreading is the first and foremost step in the interaction of cells with the complimentary materials and its growth could be affected by the nature of materials [11].

The cell proliferation capacity of MC3T3-E1 cells in $\mathrm{CH}$ and $\mathrm{CGHaP}$ microspheres were evaluated by AlamarBlue colorimetric assay. Two kinds of observations could be made from this proliferation assay. First, limited cell proliferation in case of control was attributed to the lack of surface for spreading. Second, high proliferation in case of microspheres (especially $\mathrm{HaP}$ one) was attributed to the high surface area and suitable environment. Nearly, 2-fold higher cell proliferation was observed in this case. Enhanced cytocompatibility was evident for the HaP-containing microspheres, as cells showed a faster growth rate than on bare chitosan microspheres $[12,13]$

The CGHaP microspheres showed high mineralization capacity which would be a great boon for the bone tissue engineering. Consistently, CGHaP microspheres showed a high ALP activity (2-fold) while $\mathrm{CH}$ showed lesser ALP activity after 7 days incubation. Studies have shown that HaP can stimulate the cell proliferation of human and animal osteoblasts. Thus, the active effect on the cell proliferation of the composite was mainly attributed to the biological benefits of HaP.

\section{CONCLUSION}

Chitosan/gelatin-hydroxyapatite (CGHaP) microspheres have been successfully formulated. The presence of hydroxyapatite in the microspheres remarkably promotes MC3T3-E1 cell proliferation and spread on the surface of the microspheres which paves way for facilitated bone ingrowth formation and possibility of good osteointegration. The ability of CGHaP microspheres to promote cell attachment and extracellular matrix mineralization will further facilitate bone tissue engineering. Overall, the results indicate that the presence of $\mathrm{HaP}$ in composite microspheres increases surface roughness and protein affinity, thereby enhancing biological response. The outcome of present study would have a great impact on the development of novel nanomaterials for bone tissue engineering with potential clinical applications.

\section{DECLARATIONS}

\section{Acknowledgement}

The study was funded from Technical Grant of Jining First People's Hospital, China.

\section{Conflict of Interest}

No conflict of interest associated with this work.

\section{Contribution of Authors}

The authors declare that this work was done by the authors named in this article and all liabilities pertaining to claims relating to the content of this article will be borne by them.

\section{REFERENCES}

1. Shakir M, Jolly $R$, Khan MS, Iram N, Sharma TK, AlResayes SI. Synthesis and characterization of a nanohydroxyapatite/chitosan/polyethylene glycol nanocomposite for bone tissue engineering. Polym. Adv. Technol. 2015; 26: 41-48

2. Murugan R, Ramakrishna S. Bioresorbable composite bone paste using polysaccharide based nano hydroxyapatite. Biomaterials 2004; 25: 3829-3835. 
3. Jiang $H$, Zuo $Y$, Zou $Q$, Wang $H$, Du J, Li Y, Yang $X$. Biomimetic spiral-cylindrical scaffold based on hybrid chitosan/cellulose/nano-hydroxyapatite membrane for bone regeneration. ACS Appl. Mater. Interfaces 2013; 5: 12036-12044.

4. Lei Y, Guan JJ, Chen W, Ke QF, Zhang CQ, Guo YP. Fabrication of hydroxyapatite/chitosan porous materials for $\mathrm{Pb}$ (II) removal from aqueous solution. RSC Adv. 2015; 5: 25462-25470.

5. Bansal V, Sharma PK, Sharma N, Pal OP, Malviya R. Applications of chitosan and chitosan derivatives in drug delivery. Adv. Biol. Res. 2011; 5: 28-37.

6. Bernkop-Schnürch A, Dünnhaupt S. Chitosan-based drug delivery systems. Eur. J. Pharm. Biopharm. 2012; 81: 463-469.

7. Laurencin CT, Ashe KM, Henry N, Kan HM, Lo KWH. Delivery of small molecules for bone regenerative engineering: preclinical studies and potential clinical applications. Drug Discov. Today 2014; 19: 794-800.

8. Sonia T, Sharma CP. Chitosan and its derivatives for drug delivery perspective, in: Chitosan for Biomaterials I, Springer 2011; 23-53
9. Kikuchi $M$, Itoh $S$, Ichinose $S$, Shinomiya K, Tanaka J. Self-organization mechanism in a bone-like hydroxyapatite/collagen nanocomposite synthesized in vitro and its biological reaction in vivo. Biomaterials 2001; 22: 1705.

10. Toworfe GK, Composto RJ, Shapiro IM, Ducheyne P. Nucleation and growth of calcium phosphate on amine-, carboxyl- and hydroxyl-silane self-assembled monolayers. Biomaterials 2006; 27: 631

11. Györgyey Á, Ungvári K, Kecskeméti G, Kopniczky J, Hopp B, Oszkó A, Pelsöczi I, Rakonczay Z, Nagy K, Turzó K. Attachment and proliferation of human osteoblast-like cells (MG-63) on laser-ablated titanium implant material. Mater. Sci. Eng. C Mater. Biol. Appl. 2013; 33: 4251-4259.

12. Tan H, Guo S, Yang $S, X u X$, Tang T. Physical characterization and osteogenic activity of the quaternized chitosan-loaded PMMA bone cement. Acta Biomater. 2012; 8: 2166-2174.

13. Lee M, Li W, Siu RK, Whang J, Zhang X, Soo C, Ting K, Wu B. M. Biomimetic apatite-coated alginate/chitosan microparticles as osteogenic protein carriers. Biomaterials, 2009; 30: 6094-6101 